\title{
Das Renal Cell Carcinoma Network (RCC Net)
}

\section{Sacha Rothschild,}

Frank Stenner

Universitätsspital Basel,

Medizinische Onkologie, Basel

Interessenkonflikte

SR: Honorare von Bayer, Pfizer, GlaxoSmithKline und Roche

für Advisory Boards.

FS: Honorare als Berater

für Pfizer, Bayer, GSK

Novartis und Roche.

1 nicer - Foundation National Institute for Cancer Epidemiology and Registration (database on the Internet). 2012.
Korrespondenz:

Dr. med. et Dr. phil. nat.

Sacha Rothschild

Universitätsspital Basel

Medizinische Onkologie

Petersgraben 4

CH-4031 Basel

Tel. 0612655074

Fax 0612655316

sacha.rothschild[at]usb.ch

\section{Einleitung}

Das Nierenzellkarzinom (früher Hypernephrom) ist der häufigste maligne Nierentumor und ist für ca. $85 \%$ aller Malignome in diesem Organ verantwortlich. Die Inzidenz in der Schweiz liegt bei 792 Neuerkrankungen pro Jahr, wobei Männer fast doppelt so häufig betroffen sind wie Frauen. Das Nierenzellkarzinom ist in der Schweiz für rund $2 \%$ aller krebsbedingten Todesfälle verantwortlich [1]. Das Nierenzellkarzinom ist somit eine seltene Erkrankung, weswegen es auch nur wenige Fachärzte in der Schweiz gibt, die auf die Behandlung dieser Patienten spezialisiert sind. Die Behandlung des Nierenzellkarzinoms hat sich durch das verbesserte biologische Verständnis der Erkrankung in den letzten Jahren gewandelt. Seit 2005 wurden sieben neue Medikamente zur Behandlung des fortgeschrittenen und/oder metastasierten Nierenzellkarzinoms zugelassen. Dazu kommen auch chirurgische und interventionelle Therapieoptionen. Die Behandlung von Patienten mit Nierenzellkarzinom wird dadurch zunehmend komplexer und bedarf der engen Zusammenarbeit von Spezialisten verschiedener Fachdisziplinen, unter anderem Hausärzte, Urologen, Nephrologen, Radiologen, interventionelle Radiologen, Pathologen, medizinische Onkologen und Radio-Onkologen.

\section{Organisation}

Das RCC Net ist eine unabhängige, nicht gewinnorientierte Organisation in der Rechtsform eines Vereins. Das RCC Net wird von folgenden Unter-

\section{Le Renal Cell carcinoma network (RCC Net)}

Le réseau RCC Net regroupe des médecins exerçant en Suisse et intervenant dans la prise en charge de patients atteints de carcinome rénal, avec pour objectif d'harmoniser les standards de qualité, d'améliorer la qualité du traitement, de permettre un transfert de connaissances entre les différentes disciplines impliquées dans la prise en charge de patients atteints de carcinome rénal et de participer à l'élaboration de la formation postgraduée dans ce domaine.

\section{Zielsetzungen}

Das RCC Net hat sich folgende Ziele gesetzt:

- Einen Beitrag leisten zur Verbesserung der Prävention, Diagnostik, Therapie und Nachsorge von Patienten mit Nierenzellkarzinom;

- Angebot einer schweizweiten Plattform zur Diskussion wissenschaftlicher und praxisrelevanter Aspekte zum Nierenzellkarzinom;

- Erarbeitung und Verbreitung von ausgewogenen, objektiven Informationsgrundlagen und Hilfestellungen zu diesem komplexen Krankheitsbild;

\section{Die Behandlung wird zunehmend komplexer und bedarf der engen Zusammenarbeit von Spezialisten verschiedener Fachdisziplinen.}

nehmen mit einer nicht zweckgebundenen Zuwendung unterstützt: Bayer HealthCare, GlaxoSmithKline, Novartis, Pfizer. Der Verein wird von Pro Medicus $\mathrm{GmbH}$ als Geschäftsstelle betreut.

Das RCC Net steht allen medizinischen Onkologen und anderen Fachärzten offen, die Nierenzellkarzinompatienten behandeln. Anfragen können an die Geschäftsstelle (rccnet[at]promedicus.ch) gerichtet werden. Weitere Informationen sind in den Statuten zu finden, die auf der Website www.rccnet.ch verfügbar sind.
- Etablierung des RCC Net als Berufs- und institutionenübergreifende Plattform für alle medizinischen Fachpersonen, die Nierenzellkarzinompatienten behandeln und betreuen.

\section{Website www.rccnet.ch}

Das RCC Net wurde 2011 gegründet von Ärzten verschiedener Fachdisziplinen, die in der Betreuung und Behandlung von Patienten mit Nierenzellkarzinom involviert sind. Als Plattform des Netzwerks dient die Website www.rccnet.ch. Die Website dient 


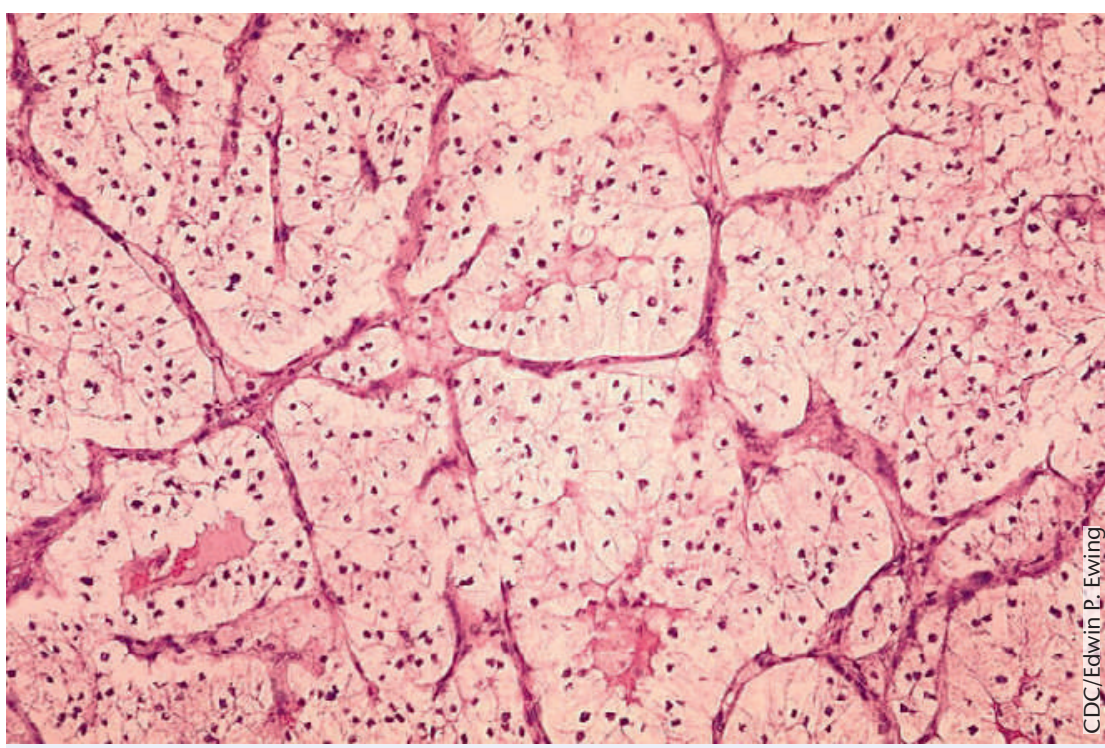

Histopathologie eines hellzelligen Nierenzellkarzinoms. der Informationsverbreitung, beinhaltet eine Literaturdatenbank mit aktuellen Artikeln aus medizinischen Zeitschriften, die von Mitgliedern des Netzwerkes verfasst wurden und ist auch die Kommunikationsplattform mit den Mitgliedern des Netzwerkes, zum Beispiel im Rahmen des neugeschaffenen virtuellen Tumorboards. lungnahme ab und stehen auch für weitere Fragen zum Fall zur Verfügung. Das Pilotprojekt unter der Leitung von PD Dr. med. F. Stenner hat im Januar 2013 gestartet und es konnten bereits mehrere Fälle diskutiert werden. Anfragen für Fallbesprechungen können gerichtet werden an tumorboard[at]rccnet.ch. Innert 24 Stunden nach Eingang der Anfrage wird mit dem behandelnden Kollegen Kontakt aufgenommen und im kurzen Intervall eine Antwort gegeben.

\section{1st Annual Meeting - interdisziplinäre Fortbildung}

Die erste Jahresversammlung des RCC Net findet am 19. September 2013 (13.30-18.30 Uhr) am Flughafen in Zürich statt und ist als interdisziplinäre Fortbildung gestaltet. Diese halbtägige Veranstaltung richtet sich an Mediziner(innen) jeglicher Fachrichtung, die sich für das Nierenzellkarzinom interessieren und/oder Nierenzellkarzinompatienten behandeln. Anerkannte Spezialisten der verschiedenen Fachrichtungen aus der Schweiz und dem Ausland werden zu Diagnose, Therapieoptionen und laufenden Forschungsprojekten berichten. Zudem sollen interessante Fälle vorgestellt und diskutiert werden. Diese Veranstaltung ist von allen involvierten Fachgesellschaften (SGIM, SGMO, SGN, SGR, SGU, SRO) anerkannt, und es werden entsprechende Fortbildungscredits vergeben. Das Programm steht auf der Website www.rccnet.ch zur Verfügung. Die Online-Anmeldung ist eben-

\section{Das RCC Net steht allen medizinischen Onkologen und anderen Fachärzten offen, die Nierenzellkarzinompatienten behandeln.}

\section{Virtuelles Tumorboard}

Das RCC Net bietet fachärztlichen Kolleginnen und Kollegen an, ihre Nierenzellkarzinompatienten Mitgliedern des RCC NET im Rahmen eines virtuellen Tumorboards vorzustellen und $\mathrm{zu}$ besprechen. Mitglieder des RCC Net besprechen die vorgestellten Fälle und geben anschliessend eine schriftliche Stel- falls auf der Website verfügbar. Teilnehmer haben auch die Möglichkeit, eigene Patientenfälle vorzustellen und mit den Referenten zu besprechen. Zwecks optimaler Vorbereitung sollten die Fälle vor dem Meeting eingereicht werden (rccnet[at] promedicus.ch). 